\title{
INATTENTIONAL BLINDNESS WHILE DRIVING
}

\author{
Chip Wood \\ Human Interface Lab-Phoenix \\ Motorola Labs \\ 7700 S. River Parkway \\ Tempe, AZ 85284 \\ E-mail: Chip.Wood@Motorola.com \\ Rob Gray \\ Dept. of Applied Psychology \\ Jennifer Young \\ Global Technology Development \\ Arizona State University East \\ John Summers, Kari Torkkola, Noel Massey \\ Human Interface Lab-Phoenix \\ Motorola Labs
}

\begin{abstract}
Mack and Rock's research (1999), suggests that we perceive only those objects and events to which we directly attend. This means that the majority of unexpected visual information goes unnoticed, no matter how dramatic or important it may be. Not noticing unexpected objects in direct view because your attention is on other driving events is a potentially deadly but common phenomenon. Most drivers have experienced these brief moments of "functional blindness" and not perceived events or objects directly and obviously centered in their field of vision. This usually produces astonishment, alarm, and possible over-reaction when awareness returns. The driver may be telling the truth to the officer after the crash, "I did not see that stop sign!"

Other experiments (Simons and Chabris, 1999) have shown that "InAttentional Blindness" is exhibited by a majority of viewers when an unexpected object and action take place clearly, slowly, and within inches of objects being attended to. Wickens, et al. (1998) examined how pilots in flight simulators perform using head-up displays. The research showed that when experimenters put something unexpected, but important, in pilots' field of vision, such as an airplane on the runway, pilots often land right on top of them.
\end{abstract}

This paper will describe what we believe to be the first experiment explicitly designed to test this phenomenon while driving in a simulator. Our study is unlike the normal driver distraction study in that we did not ask the driver to divert attention to an off-the-road secondary task, but to keep attention focused solely on the road and the objects on it.

Subjects drove on a two-lane road in a city environment generated by a 4-channel Class II GlobalSim driving simulator. We manipulated drivers' attention by asking them to count the number of a specific type of pedestrian randomly interspersed with other pedestrians strolling along the right side of the road. Meanwhile various expected and unexpected critical driving 
events - stop signs at intersections (in the line of pedestrians), random lead car braking, and barriers in the roadway - were presented. The reaction times, as sensed by brake pedal pressure, to the critical driving events were recorded and compared to a baseline condition where the drivers were asked to merely follow normal driving procedures.

Compared to the baseline, the pedestrian-monitoring task increased reaction times to all types of critical driving events. Subjective observations of drivers and implications for road safety will be discussed.

\section{References}

Mack, A. \& Rock, I. (1999). Inattentional Blindness. Psyche, Cambridge, MA:MIT.

Simons, D.J., \& Chabris, C.F. (1999). Gorillas in our midst: Sustained inattentional blindness for dynamic events. Perception, 28, 1059-1074.

Wickens, Gordon, Liu. (1998). An Introduction to Human Factors Engineering. New York: Addison-Wesley-Longman. 